\title{
Automated Detection of Fetal Movements Based on Baseline Calculation and Fetal Heart Rate Accelerations
}

\author{
Mei-Jia Huang ${ }^{1} \&$ Hui-Jin Wang ${ }^{1}$ \\ ${ }^{1}$ School of Information Science and Technology, Jinan University, Guangzhou, China \\ Correspondence: Hui-Jin Wang, School of Information and Technology, Jinan University, Guangzhou, China. E- \\ mail: twanghj@jnu.edu.cn
}

Received: July 9, 2018

Accepted: July 19, $2018 \quad$ Online Published: August 27, 2018

doi:10.5539/mas.v12n9p173

URL: https://doi.org/10.5539/mas.v12n9p173

\begin{abstract}
Fetal electronic monitoring is extensive and important in obstetrics. Although fetal movement is ususally used as an important indicator for quantifying fetal wellbeing, non-invasive and long-term monitoring of fetal movement remains challenging. The object of this study is to develop an algorithm for automatic detection of the fetal movements based on the analysis of Doppler ultrasound signals. In order to detect fetal movements automatically, a two-step process was proposed to track fetal movement. In Step 1, to suppress the problem of error detection, we calculated the baseline of the fetal movement signals from actography to extract new signals. In step2, we recalculated the threshold value of fetal movement detection by utilizing the information of fetal heart rate (FHR) acceleration to produced adaptive threshold values. The results showed that the union of results detected by the proposed method from actography and tocography achieved an encouraging performance with highest sensitivity and acceptable positive predictive value (PPV).
\end{abstract}

Keywords: fetal movement, baseline, fetal heart rate acceleration

\section{Introduction}

Fetal movement has been used as an indicator of fetal health for a long time (Sadovsky \& Yaffe, 1973; Sadovsky, Mahler, Polishuk, \& Malkin, 1973). Studying in maternal fetal movement counts as an indicator of fetal health began to flourish in the 1970s and 1980s (Pearson, 1979; Wilailak, Suthutvoravut, Cherng-Sa-Ad, Herabutya, \& Chaturachinda, 1992). It has been indicated that fetal movement reduction is associated with adverse pregnancy outcomes, both in high-risk and low-risk pregnancies and increasing awareness of maternal sensorimotor activity can reduce the rate of stillbirth (Frøen, 2004). Even with various fetal assessment methods to determine optimal delivery time, fetal movement is still a component of clinical decision-making (Lai, Nowlan, Vaidyanathan, Shaw, \& Lees, 2016). The methods of determining fetal movement generally include maternal involvement, clinical involvement, technical assistance and automation technology (Stanger, Horey, Hooker, \& Custovic, 2017). In automation technology field, the signal called actogram has become an integral part of the cardiotocography trace providing information on the fetal movement activity (Wróbel et al., 2014).

In the study of the algorithm of ultrasonic Doppler fetal movement, Maeda et al. (1988) used the 20-80hz bandpass filter to filter the Doppler frequency shift signals, and then processed the signals and shifted the signals to display signals. Shinozuka and Yamakoshi (1993) used multichannel ultrasonic pulse Doppler to calculate the internal tissue displacement method, and the maximum entropy method was used to identify fetal movement. Pourcelot et al (1998) studied the calculation of fetal dynamic displacement with the analytic signals of non-directional Doppler audio signals, and then combined the methods of time domain and frequency domain analysis to separate fetal movement signals. All of these methods above focused more on signal acquisition and improving the signal quality. Lu et al. (2014) used Ostu method to obtain the fixed threshold automatically for fetal movement detection, which focused on the methodology of detection from actography. However, fixed threshold cannot adapt to local variation of fetal movement signals.

On the other hand, time relation between fetal heart rate (FHR) acceleration and fetal movement has been confirmed (Timor-Tritsch et al., 1978; Dipietro et al., 1996; Zhao \& Wakai., 2002; Maeda, 2015), and the acceleration of fetal heart rate with fetal movement is considered as a manifestation of fetal health (Dipietro, 1996; Bocking, 2003). Thus, to overcome the shortcoming of fixed threshold and achieve better adaptability, we propose a two-step procedure process. The first step involves a baseline calculation method for fetal movement signals 
from actography to cut down the detection faults. The second step involves using the information of fetal heart rate accelerations as basic to recalculate the threshold value of fetal movement signals. The performance of the method proposed are evaluated by the sensitivity and positive predictive value (PPV) in experimental.

\section{Method}

\subsection{Acquisition and Preprocessing}

The experimental data cotains 42 pairs of signals selected from the clinical data from the People's Hospital of Baiyun District, Guangzhou (Lu et al., 2014), including antenatal FHR, TOCO and fetal movement tracing. Each signal contains more than 20 minutes of monitoring data. The fetal movements were recorded by an experienced obstetric expert with more than 30 years of experience manually.

To detect the FHR heart accelerations, we processed the abnormal data which contained missing points or pseudoimages. The breakpoints were filled with the linear interpolation method. For fetal tracking, the background noise was further suppressed by bandpass filter. Then the fetal movement signals were de-noise by a wavelet analysis algorithm.

Unlike the actual fetal movement signals, TOCO signals consisting of uterine contraction signals were weak and undetectable. To extract fetal movement signals, the original contraction data were smoothed through the 31-point Hanning window, and the amplitude amplification and time deviation generated by smoothing processing were corrected to obtain the smooth signals defined as sequence Filtereduc ${ }_{i}$. Next we subtracted the original contractions from Filtereduc $c_{i}$ and get the difference sequence defined as TocoFmSignal $_{i}, i=1$ to $\mathrm{N}$, which

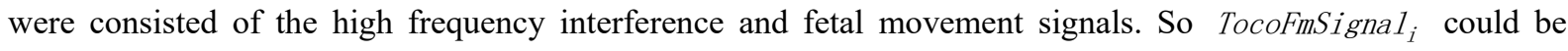
regarded as the signals for fetal movement identification.

\subsection{Automated Detection of Fetal Movements}

\subsubsection{Ostu Method}

The Ostu method (Otsu, 2007) is used to set the threshold to distinguish between fetal movements and background noise automatically. The signals above the threshold are regarded as fetal movements and the rest are background noise. Let $T$ denote the hypothetical threshold in the Ostu method.

Compared with the threshold, the ratio of the signals that less than or equal to the threshold $T$ is defined as $\omega_{0}$, and the average value of these signals is defined as $u_{0}$. The ratio of the signal greater than $T$ to the total signal is defined as $\omega_{1}$ and the average of these signals is defined as $u_{1}$.

Then, the average of the whole signals can be represented by:

$$
\mathrm{u}=\omega_{0} u_{0}+\omega_{1} u_{1}
$$

The criterion for measuring the difference in the Ostu algorithm is the inter-class variance. So the inter-class variance can be defined as:

$$
\mathrm{g}=\omega_{0}\left(u_{0}-u\right)^{2}+\omega_{1}\left(u_{1}-u\right)^{2}=\omega_{0} \omega_{1}\left(u_{0}-u_{1}\right)^{2}
$$

When $g$ reaches the maximum, the maximum difference between two parts of the signal is expressed, and the corresponding threshold $T$ can be regarded as the optimal threshold. The signals above the optimum threshold $T$ are considered to be fetal movements in this study. The Ostu method was marked as Alg1 in the experiment.

As shown in Figure 1, the threshold of fetal movement obtained by the Otsu method performs well when signals are relatively clear and stable, but it is less effective for the situation where the local signal fluctuation is large. It can be seen that there are lots of false detections in the area where the fetal movements are marked in red box.

\subsubsection{Fetal Movement Signals Extraction by Baseline from Actography}

The phenomenon of large fluctuation in the local signal is often manifested in the overall elevation of signals, which cannot be effectively identified by the conventional threshold method. Here, we solve this problem by calculating the fetal movement baseline.

Baseline is often used in fetal heart rate as the basis for interpretation of variation, acceleration and deceleration of FHR models. Comparing the fetal movement signals with the baseline can improve the inaccuracy of recognition when the local signal instability occurs.

Based on Andersson's estimation method for fetal heart rate baseline, histogram analysis and filtering are applied to baseline calculation, and the method of fetal movement curve is modified and simplified here. 


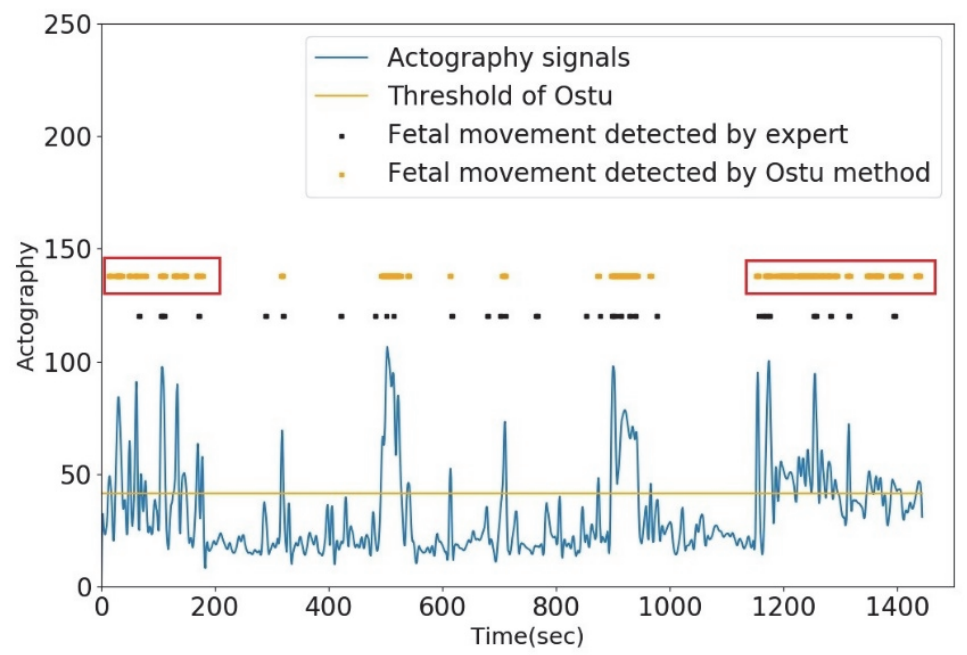

Figure 1 . The fetal movement detections by Ostu method from actography

Let the sequence ActoSignal ${ }_{\mathrm{i}}, i=1$ to $N$ denote the fetal movement signals from actography. Let the sequence $A c t o B$ aseli $n e_{\mathrm{i}}, i=1$ to $N$ denote the baseline of ActoSignal. To calculate the baseline of fetal movement we use the following steps:

First we calculate the average value of the first $15 \%$ of the value of signals in ActoSignal $I_{\mathrm{i}}$ per minute as the initial reference value ActoBase.

Then using the following method to trim ActoSignal:

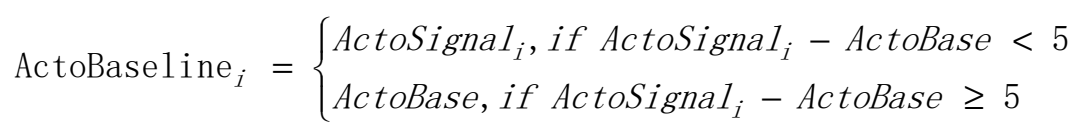

Next filtering ActoBaseline backwards and forwards. The forward filtering formula is identified as follows:

$$
\text { ActoBaseli ne }_{i}=0.8 \text { A }_{\text {toBaseline }} \text { t }_{1}+0.2 \text { ActoBaseli ne }_{i}
$$

The backward filtering formula is identified as follows:

$$
\text { ActoBaseli ne }_{i}=0.8 \text { A }_{\text {toBaseline }}+1+0.2 \text { ActoBaseli ne }_{i}
$$

Different from the baseline of fetal heart rate, the baseline of fetal movement only requires the trend of the lower part of fetal movement signals. Here, the baseline of fetal movement can meet the demand after two times of forward and backward filtering.

So the new fetal movement signals ActoDiffSi gnal $1_{\mathrm{i}}$ is identified as follows:

$$
\begin{gathered}
\text { ActoDiff }_{i}=\text { ActoSignal }_{i}-\text { ActoBaseline }_{i} \\
\text { ActoDiffSignal }_{i}=\left\{\begin{array}{l}
\text { ActoDiff }_{i}, \text { if ActoDiff } \\
0, \text { if ActoDiff }
\end{array}<0\right.
\end{gathered}
$$

Figure 2 shows the baseline obtained by the proposed method above from the signals in Figure1. And figure 3 shows the fetal movement signals extraction by using baseline from actography and the fetal movement detections

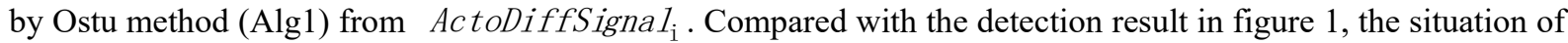
undue-detection marked in red box had been improved. 


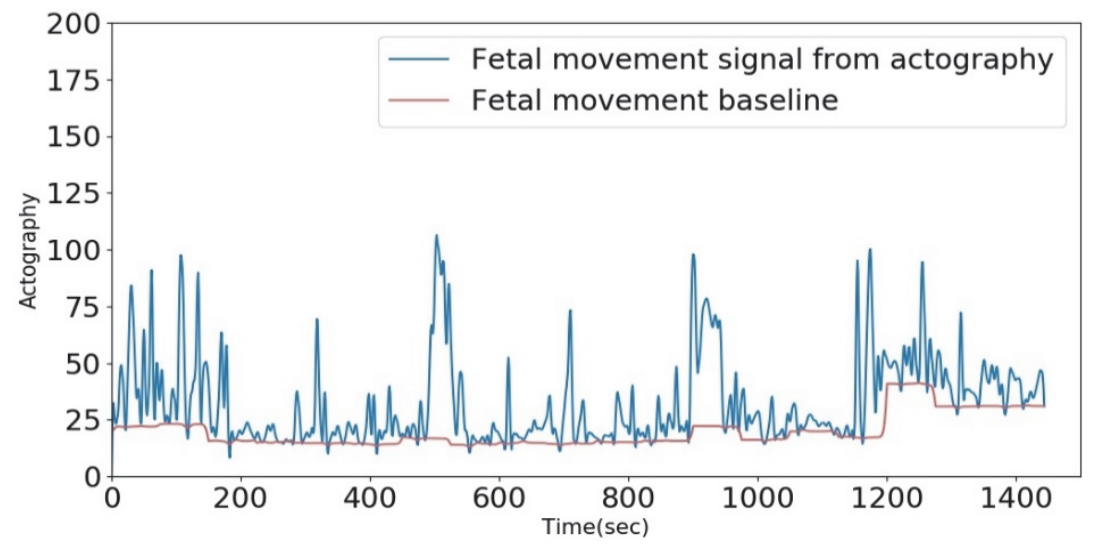

Figure 2. The fetal movement baseline obtained by proposed method

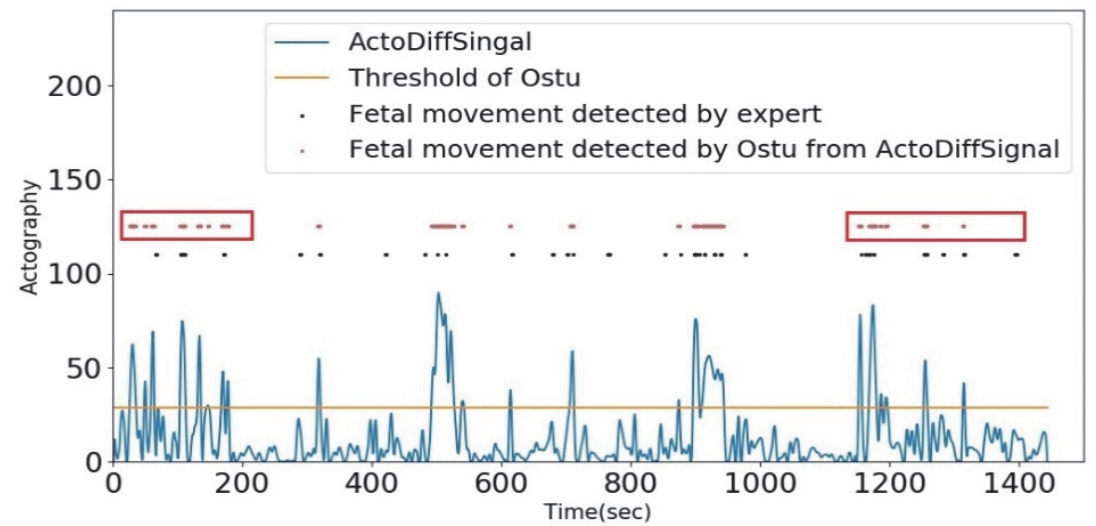

Figure 3. The fetal movement signals ActoDiffSignal extraction by baseline from actography and the fetal movement detections by Ostu method from ActoDiffSignal

\subsubsection{Threshold Recalculation for fetal heart Rate Acceleration Interval}

Due to the linkage relationship between fetal movement and acceleration, the information of FHR accelerations can be used as the assistant identification of identifying fetal movements.

Let the sequence $A c c_{i}, i=1$ to $N$ denote the FHR acceleration points detected by Andersson's estimation method (Andersson, 2011), where $\mathrm{N}$ is the total number of FHR acceleration points. For fetal movement signals $\mathrm{Sig}_{i}$, let $\operatorname{inAccSeg}(i)$ denote if there exists a point of FHR acceleration $A c c_{i}$ within one minute at the time of the $\operatorname{Sig}_{i}$, and if it exists, $\operatorname{inAccSeg}(i)=1$, vice versa, $\operatorname{inAccSeg}(i)=0$. And we define $\operatorname{AccSeg}(i)$ as the fetal movement signal segment which are within one minute before and after the time of $A c c_{i}$ above.

We define the method for obtaining threshold value by Ostu method as $T$ (SignalSeg), where SignalSeg is the segment of fetal movement signals. With the acceleration of fetal heart rate, the thresholds of fetal movement within the range of the acceleration segments are recalculated.

So let the sequence $N T_{\mathrm{i}}, i=1$ to $N$ denote the threshold of fetal movement detection, where $\mathrm{N}$ is the length of fetal movement signals. $N T_{\mathrm{i}}$ is calculated as follows:

$$
\mathrm{NT}_{\mathrm{i}}=\left\{\begin{array}{l}
T, \text { if } \operatorname{inAccSeg}(i)=0 \\
T(\operatorname{AccSeg}(i)), \text { if } \operatorname{inAccSeg}(i)=1
\end{array}\right.
$$

Because the threshold obtained by narrowing the length of Ostu may be not ideal, we integrate the whole and local signal situation by adding weights to adjust threshold values. Thus $N T_{\mathrm{i}}$ is revised as follows:

$$
\mathrm{NT}_{\mathrm{i}}=\left\{\begin{array}{l}
T, \text { if } \operatorname{isAcc}(i)=0 \\
\alpha \cdot\left(\omega_{1} \cdot T+\omega_{2} \cdot T(\operatorname{AccSeg}(i))\right), \text { if } \operatorname{isAcc}(i)=1
\end{array}\right.
$$

Where $\alpha$ is the adjustment coefficient, $\omega_{1}$ and $\omega_{2}$ are the weights assigned to threshold values. Let $\alpha=0.8, \omega_{1}=0.2$ and $\omega_{2}=0.8$, which can achieved fine results through tests. 
And this method was marked as Alg2 in the experiment.

Figure 4 shows the result of fetal movements detected by Alg1 and Alg2 from ActoDiffSi gnal $1_{i}$ of a sample. In Alg2, the threshold values in the range of $\operatorname{AccSeg}(i)$ (marked in green background) are recalculated and fetal movements undetected by Alg1 can be detected by Alg2 which are marked in red box.
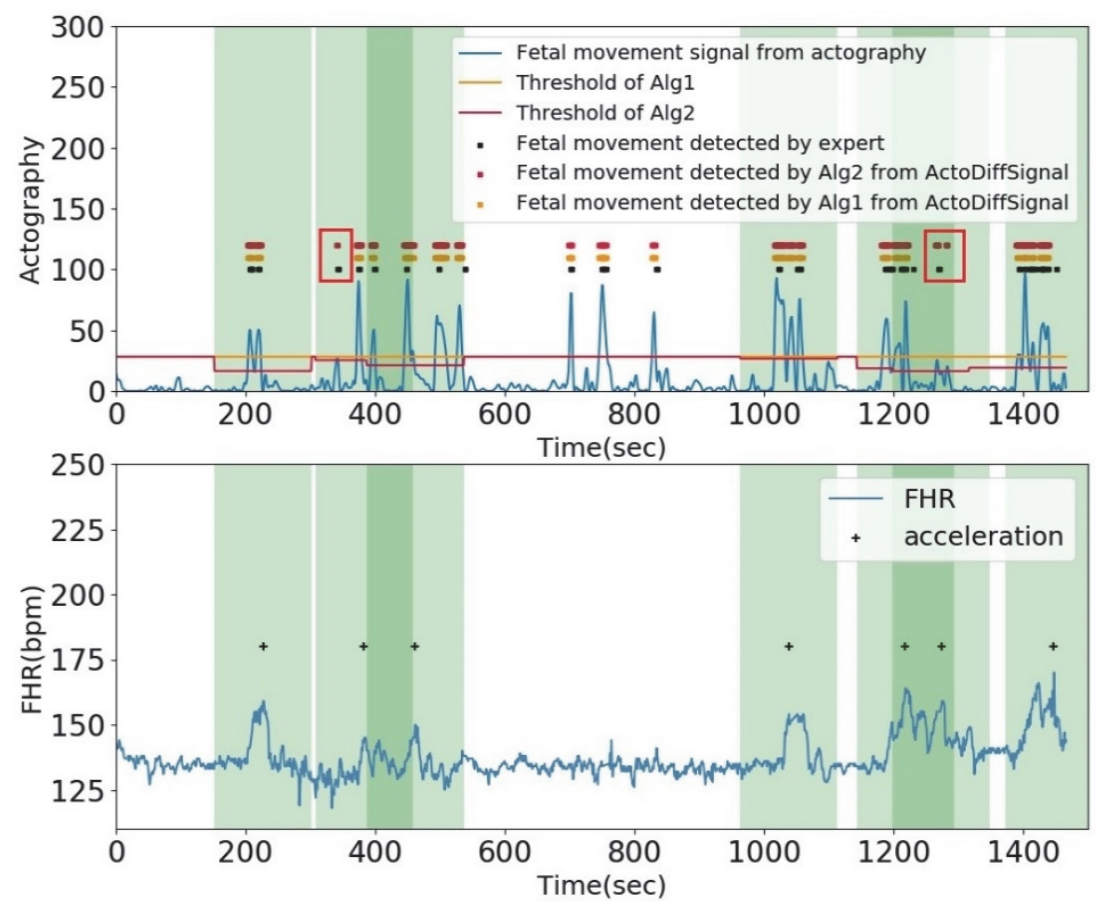

Figure 4. The result of fetal movement detected by Alg1 and Alg2 from ActoDiffSignal

\section{Results and Discussion}

The fetal movement detected by expert manually has time-skew errors. In order to assess the accuracy of the automatic movement detection, the fetal movements detected by algorithms are considered correct when it is in the time range within $-3 \mathrm{~s} \sim 3 \mathrm{~s}$ of the fetal movement detected by the expert.

For convenience, fetal movements detected by expert are called expert-detected movements and fetal movements detected by algorithms are called automatic movements. Since the position of the automatic movement position may be close to the position of multiple expert-detected movements, which means an automatic fetal movement corresponds to multiple expert-detected movement positions, we sign expert-detected movement which can be found in certain time range (3s) of one of automatic movements. So the sensitivity is defined as the ratio of the expert fetal movements signed by the automatic movements to the total expert-detected movements, not the ratio of correct automatic movements to the total number of expert-detected movements. And the positive predictive value (PPV) is the ratio of correct automatic movements to total automatic fetal movements.

In the experiments, Alg1 and Alg2 were applied to the fetal movement signals from actography and tocography, and the sensitivity and PPV of two algorithms were calculated to evaluate their performance. The union of results of actography and tocography were also included in the assessment.

Table I shows that Alg2 performs better than Alg1 both in sensitivity and PPV from different source data of fetal movements. Compared with Alg1, the increasing amplitude of sensitivity of Alg2 from ActoSignal ${ }_{\mathrm{i}}$ and ActoDiffSignal $I_{\mathrm{i}}$ is not evident. After observing the recognition results we found that Alg2 could detect a part of fetal movements that undetected by Alg1. But due to a large fetal movement base, the value of sensitivity of Alg2 do not increase apparently. 
Table 1. PERFORMANCE COMPARISON

\begin{tabular}{|c|c|c|c|c|c|}
\hline Data & Method & $\mathrm{CE} / \mathrm{E}$ & Sensitivity & $\mathrm{CA} / \mathrm{A}$ & PPV \\
\hline \multirow{2}{*}{ TocoFmSignal $_{i}$} & Alg1 & $1216 / 3146$ & 0.3865 & $752 / 2204$ & 0.3412 \\
\hline & Alg2 & $1266 / 3146$ & 0.4024 & $782 / 2265$ & 0.3453 \\
\hline \multirow{2}{*}{ ActoSignal $_{\mathrm{i}}$} & Alg1 & $2310 / 3146$ & 0.7343 & $2106 / 3749$ & 0.5617 \\
\hline & $\operatorname{Alg} 2$ & $2320 / 3146$ & 0.7374 & $2114 / 3762$ & 0.5619 \\
\hline \multirow{2}{*}{ TocoFmSingal $_{i}$ ActoSignal $_{\mathrm{i}}$} & Union of Alg1 & $2532 / 3146$ & 0.8048 & $2858 / 5953$ & 0.4801 \\
\hline & Union of Alg2 & $2546 / 3146$ & 0.8093 & $2896 / 6027$ & 0.4805 \\
\hline \multirow{2}{*}{ ActoDiffSignal $_{\mathrm{i}}$} & Alg1 & $2219 / 3146$ & 0.7053 & $1920 / 3284$ & 0.5847 \\
\hline & Alg2 & $2226 / 3146$ & 0.7076 & $1928 / 3291$ & 0.5858 \\
\hline \multirow{2}{*}{ TocoFmSinga $_{i}$, ActoDiffSignal $l_{\mathrm{i}}$} & Union of Alg1 & $2487 / 3146$ & 0.7905 & $2672 / 5488$ & 0.4869 \\
\hline & Union of Alg2 & $2500 / 3146$ & 0.7947 & $2710 / 5557$ & 0.4877 \\
\hline
\end{tabular}

Note: Statistics evaluated using patterns: CE represents the numbers of expert fetal movements signed by the automatic fetal movements, E represents the number of the total expert fetal movements. CA represents the number of correct automatic fetal movements and A represents the number of total automatic fetal movements.

Compared with the detection result from ActoSignal $1_{i}$, the sensitivity of ActoDiffSignal by both Alg1 and Alg2 appears a decreasing trend which is acceptable. Different from ActoSignal $I_{\mathrm{i}}$, ActoDiffSignal $\mathrm{I}_{\mathrm{i}}$ aims to reduce the error detection of the signals which has large fluctuations and at the same time it will reduce the number of fetal movements detected by algorithms, which may cause a slight decrease of sensitivity. However the reduction of fetal movements increases PPV which is identical to the expectation.

On the other hand, both sensitivity and PPV obtained from TocoFmSignal ${ }_{i}$ are lower than $40 \%$. But higher sensitivity and acceptable PPV are obtained by combining the detection result of TocoFmSignal $_{i}$ with ActoSignal or ActoDiffSignal $_{\mathrm{i}} .80 .83 \%$ of sensitivity and $48.10 \%$ of PPV are obtained by Alg2 algorithm by the combination of TocoFmSign al ${ }_{i}$ and ActoSignal ${ }_{i}$, and $79.37 \%$ of sensitivity and $48.75 \%$ of PPV were obtained by the combination of TocoFmSignal ${ }_{i}$ and ActoDiffSignal $I_{i}$, which indicates that TocoFmSignal Can $_{i}$ be regard as auxiliary source data for fetal movement.

Since the pregnant woman can only perceive about 30 percent of the fetal movements, the fetal movements detected by the algorithm have no equivalent in the maternal markers by expert, and may actually represent real fetal movements, which means that our algorithm can achieve higher sensitivity and PPV in reality. Conclusively it is recommended to use the union detection results from ActoDiffSignal $\mathrm{I}_{\mathrm{i}}$ and TocoFmSignal $_{i}$ detected by Alg2 because it had the highest sensitivity and acceptable PPV.

\section{Conclusion and Future Work}

In this study, a method for calculating the baseline of fetal movement signals from Actography was proposed to reduce the error detection of fetal movements. Subsequently, a new approach to calculate threshold values for the detection of fetal movements based on the information of fetal heart rate acceleration and Ostu method was proposed. It is proved by experiments that the proposed approach was effective and feasible. It is recommended to use Alg2 (the new approach proposed) to detect fetal movements and the union of the detection result from actography ( ActoDiffSignal $_{\mathrm{i}}$ ) and tocography $\left(\right.$ TocoFmSignal $_{i}$ ) can achieve better sensitivity and acceptable PPV.

There will be two further studies in the future:

(1) To improve the combination method by using more stable and high-quality fetal signals as identification data, and optimize the algorithms in the situation of local complex signals.

(2) To optimize the dynamic threshold method, add more analysis parameters, such as local signal quality and the shape of signal peak as the auxiliary judgment.

\section{Acknowledgments}

This work was supported by Science and Technology Planning Project of Guangdong Province, China (No.2014B010103004, No.2014B0505005011)

\section{References}

Andersson, S. (2011). Acceleration and deceleration and baseline estimation.

B., Pourcelot, D., Helgason, T., Pourcelot, L., \& Berson, M. (1998). Distance of foetal movement measured using the analytical signal derived from non-directional doppler sound. Medical Engineering \& Physics, 20(5), 325. 
https://doi.org/10.1016/S1350-4533(98)00033-2

Bocking, A. D. (2003). Assessment of fetal heart rate and fetal movements in detecting oxygen deprivation inutero. European Journal of Obstetrics \& Gynecology, 110(1), S108-S112. https://doi.org/10.1016/S03012115(03)00180-5

Dipietro, J. A., Hodgson, D. M., Costigan, K. A., Hilton, S. C., \& Johnson, T. R. B. (1996). Development of fetal movement--fetal heart rate coupling from 20 weeks through term. Early Human Development, 44(2), 139151. https://doi.org/10.1016/0378-3782(95)01704-6

Frøen, J. F. (2004). A kick from within--fetal movement counting and the cancelled progress in antenatal care. Journal of Perinatal Medicine, 32(1), 13. https://doi.org/10.1515/JPM.2004.003

Lai, J., Nowlan, N., Vaidyanathan, R., Shaw, C. J., \& Lees, C. C. (2016). Fetal movements as a predictor of health. Acta Obstet Gynecol Scand, 95(9), 968. https://doi.org/10.1111/aogs.12944

Lu, Y., Li, X., Wei, S., \& Liu, X. (2014). Fetal heart rate baseline estimation with analysis of fetal movement signal. Bio-Medical Materials and Engineering, 24(6), 3763-3769. https://doi.org/10.3233/BME-141205

Maeda, K. (2015). Invention of ultrasonic doppler fetal actocardiograph and continuous recording of fetal movements. Journal of Obstetrics \& Gynaecology Research, 42(1), 5-10. https://doi.org/10.1111/jog.12855

Maeda, K., Tatsumura, M., Nakajima, K., Ida, T., Nagata, N., \& Minagawa, Y. (1988). The ultrasonic doppler fetal actocardiogram and its computer processing. Journal of Perinatal Medicine, 16(4), 327. https://doi.org/10.1515/jpme.1988.16.4.327

Otsu, N. (2007). A threshold selection method from gray-level histograms. IEEE Trans.syst.man. \& Cybern, 9(1), 62-66. https://doi.org/10.1109/TSMC.1979.4310076

Pearson, J. F. (1979). Fetal movement recording: a guide to fetal well-being. Nursing Times, 75(38), 1639.

Sadovsky, E., \& Yaffe, H. (1973). Daily fetal movement recording and fetal prognosis. Obstetrics \& Gynecology, 41(6), 845-850.

Sadovsky, E., Mahler, Y., Polishuk, W. Z., \& Malkin, A. (1973). Correlation between electromagnetic recording and maternal assessment of fetal movement. Lancet, 1(7813), 1141. https://doi.org/10.1016/S01406736(73)91144-6

Shinozuka, N., \& Yamakoshi, Y. (1993). Measurement of fetal movements using multichannel ultrasound pulsed doppler: autorecognition of fetal movements by maximum entropy method. Medical \& Biological Engineering \& Computing, 31(1), S59-S66. https://doi.org/10.1007/BF02446651

Stanger, J. J., Horey, D., Hooker, L., \& Custovic, E. (2017). Fetal movement measurement and technology: A narrative review. IEEE Access, PP(99), 1-1. https://doi.org/10.1109/ACCESS.2017.2716964

Timor-Tritsch, I. E., Dierker, L. J., Zador, I., Hertz, R. H., \& Rosen, M. G. (1978). Fetal movements associated with fetal heart rate accelerations and decelerations. American Journal of Obstetrics \& Gynecology, 131(3), 276-280. https://doi.org/10.1016/0002-9378(78)90600-2

Wilailak, S., Suthutvoravut, S., Cherng-Sa-Ad, P., Herabutya, Y., \& Chaturachinda, K. (1992). Assessment of fetal well-being: fetal movement count versus non stress test. International Journal of Gynaecology \& Obstetrics the Official Organ of the International Federation of Gynaecology \& Obstetrics, 39(1), 23. https://doi.org/10.1016/0020-7292(92)90775-E

Wróbel, J., Kupka, T., Horoba, K., Matonia, A., Roj, D., \& Jeżewski, J. (2014). Automated detection of fetal movements in doppler ultrasound signals versus maternal perception. Journal of Medical Informatics \& Technologies, 23, 43-50.

Zhao, H., \& Wakai, R. T. (2002). Simultaneity of foetal heart rate acceleration and foetal trunk movement determined by foetal magnetocardiogram actocardiography. Physics in Medicine \& Biology, 47(5), 839. https://doi.org/10.1088/0031-9155/47/5/310

\section{Copyrights}

Copyright for this article is retained by the author(s), with first publication rights granted to the journal.

This is an open-access article distributed under the terms and conditions of the Creative Commons Attribution license (http://creativecommons.org/licenses/by/4.0/). 